\title{
Combined Breast Reduction Augmentation
}

\author{
Paul E. Chasan ${ }^{1}$ (D)
}

Received: 7 October 2019/Accepted: 8 October 2019/Published online: 15 October 2019

(C) Springer Science+Business Media, LLC, part of Springer Nature and International Society of Aesthetic Plastic Surgery 2019

No Level Assigned This journal requires that authors assign a level of evidence to each article. For a full description of these Evidence-Based Medicine ratings, please refer to the Table of Contents or the online Instructions to Authors www.springer.com/00266.

\section{Dear Editor,}

I read with interest the recent original article published in your journal titled "Combined Breast Reduction Augmentation," by Manero and Rodriguez-Vega [1]. I want to congratulate the authors both for the excellent results depicted and for the low complication rate they have achieved using their combined breast reduction acugmentation (CBRA) technique. The procedure is similar to the "reductive augmentation" technique that I published in your journal in June 2018 [2], in that both techniques address the shortcomings of conventional breast reduction (i.e., lack of superior pole fullness and bottoming out) by combining reduction with simultaneous placement of submuscular silicone gel prostheses.

There are a few substantive differences in our respective approaches upon which I wish to comment. First, the authors employ a premarked Weiss pattern in performing their reduction, whereas in all but the largest resections, we perform a circumvertical mastopexy. I find that tailortacking the breast envelope on the table as part of my circumvertical mastopexy allows for a shorter horizontal excision, whereas the Weiss pattern commits the patient to a full anchor-shaped scar at the inframammary fold. Second, in CBRA the resection is completed prior to placement of the implant into the pocket, whereas I place the

Paul E. Chasan

pchasan@drchasan.com

1 Del Mar, CA, USA implant before resection. I find that placing the implant in the pocket after the resection makes it more difficult to place the implant, and oftentimes, there are small tears or thinness at the inferior pole of the pocket.

Finally, low capsular contracture rates were reported with both procedures. Since both approaches involve large open resections of breast tissue with endless possibilities for microbial contamination, this may be of particular interest in the current debate over the role of biofilm in the development of capsular contracture.

In conclusion, I believe these two slightly different techniques, developed independently, approach both the best solution to achieving upper pole fullness and improvement in the appearance of breasts for those patients who would otherwise be candidates for breast reduction.

\section{Compliance with Ethical Standards}

Conflict of interest The author declares that he has no conflicts of interest to disclose.

Human and Animal Rights This article does not contain any studies with human participants or animals performed by the author.

Informed Consent For this type of study, informed consent is not required.

\section{References}

1. Manero I, Rodriguez-Vega A (2019) Combined breast reduction augmentation. Aesthetic Plast Surg 43:571-581

2. Chasan PE (2018) Reductive augmentation of the breast. Aesthetic Plast Surg 42:662-671

Publisher's Note Springer Nature remains neutral with regard to jurisdictional claims in published maps and institutional affiliations. 